All authors: American Cancer Society, Atlanta, GA.

Published online ahead of print at www.jco.org on September 19, 2016.

Supported by the American Cancer Society, which funded the creation and maintenance of the Cancer Prevention Study II cohort.

Authors' disclosures of potential conflicts of interest are found in the article online at www.jco.org. Author contributions are found at the end of this article.

Corresponding author: Eric J. Jacobs, $\mathrm{PhD}$, Epidemiology Research Program, American Cancer Society, National Home Office, 250 Williams St, Atlanta, GA 30303-1002; e-mail: Eric.Jacobs@cancer. org.

C 2016 by American Society of Clinical Oncology

0732-183X/16/3432w-3880w/\$20.00

DOI: 10.1200/JCO.2015.66.2361

\section{Vasectomy and Prostate Cancer Incidence and Mortality in a Large US Cohort}

Eric J. Jacobs, Rebecca L. Anderson, Victoria L. Stevens, Christina C. Newton, Ted Gansler, and Susan M. Gapstur

\title{
Purpose
}

In a recent large prospective study, vasectomy was associated with modestly higher risk of prostate cancer, especially high-grade and lethal prostate cancer. However, evidence from prospective studies remains limited. Therefore, we assessed the associations of vasectomy with prostate cancer incidence and mortality in a large cohort in the United States.

\section{Patients and Methods}

We examined the association between vasectomy and prostate cancer mortality among 363,726 men in the Cancer Prevention Study II (CPS-II) cohort, of whom 7,451 died as a result of prostate cancer during follow-up from 1982 to 2012. We also examined the association between vasectomy and prostate cancer incidence among 66,542 men in the CPS-II Nutrition Cohort, a subgroup of the CPS-II cohort, of whom 9,133 were diagnosed with prostate cancer during follow-up from 1992 to 2011. Cox proportional hazards regression modeling was used to estimate multivariable-adjusted hazard ratios (HRs) and $95 \% \mathrm{Cls}$.

\section{Results}

In the CPS-II cohort, vasectomy was not associated with prostate cancer mortality (HR, 1.01; 95\% $\mathrm{Cl}, 0.93$ to 1.10). In the CPS-II Nutrition Cohort, vasectomy was not associated with either overall prostate cancer incidence (HR, 1.02; $95 \% \mathrm{Cl}, 0.96$ to 1.08) or high-grade prostate cancer incidence (HR, $0.91 ; 95 \% \mathrm{Cl}, 0.78$ to 1.07 for cancers with Gleason score $\geq 8$ ).

\section{Conclusion}

Results from these large prospective cohorts do not support associations of vasectomy with either prostate cancer incidence or prostate cancer mortality.

\section{J Clin Oncol 34:3880-3885. (c) 2016 by American Society of Clinical Oncology}

\section{INTRODUCTION}

Vasectomy is a common, permanent, and highly effective method of birth control. ${ }^{1}$ It is less expensive and causes fewer complications than tubal ligation, the equivalent surgical sterilization procedure in women. ${ }^{1}$ Epidemiologic studies of vasectomy and risk of prostate cancer have yielded mixed results. Positive associations were observed in some hospital-based case-control studies but not in most population-based case-control studies. $^{2}$ Evidence from prospective studies is limited. Of six prospective studies published before 2014, three ${ }^{3-5}$ found a statistically significant positive association with risk of prostate cancer, but the other three ${ }^{6-8}$ found no association. All of these prospective studies were relatively small. The largest, an analysis of the Health Professionals Follow-Up Study (HPFS), ${ }^{3}$ included only 75 prostate cancer cases in vasectomized men. In 2014, an updated analysis from the HPFS was published, with more than 6,000 incident prostate cancer cases, including 1,524 in vasectomized men. ${ }^{9}$ In that analysis, by far the largest previously published, vasectomy was associated with statistically significantly higher risk of prostate cancer, including approximately $10 \%$ higher overall risk of prostate cancer and approximately $20 \%$ higher risk of high-grade (Gleason score 8 to 10) and lethal prostate cancer.

Biologic mechanisms through which vasectomy might influence prostate carcinogenesis include increased cell proliferation, which has been observed in vasectomized rats, ${ }^{10}$ as well as possible hormonal and immunologic effects of vasectomy. ${ }^{11}$ However, evidence supporting these mechanisms is limited. ${ }^{11,12}$

It is important to clarify whether vasectomy is associated with risk of prostate cancer, 
particularly with risk of more serious and fatal prostate cancers, to make well-informed choices about long-term methods of birth control. Clinical guidelines from the American Urological Association $^{1}$ state that vasectomy is not a risk factor for prostate cancer, and therefore, clinicians need not discuss risk of prostate cancer in prevasectomy counseling. However, prostate cancer is a leading cause of cancer death in men. If vasectomy increases risk of fatal prostate cancer, as suggested by results from the HPFS, this increase in risk would warrant consideration when choosing a method of birth control. Alternatively, if vasectomy does not increase risk of prostate cancer, concerns about prostate cancer should not discourage couples from choosing it.

We examined the associations of vasectomy with both prostate cancer mortality and prostate cancer incidence. The Cancer Prevention Study-II (CPS-II) is well suited to examine these associations because of its prospective design and unusually large size.

\section{PATIENTS AND METHODS}

Analyses of prostate cancer mortality were conducted in the CPS-II cohort, ${ }^{13}$ which was enrolled in 1982 and is observed only for mortality outcomes. Analyses of prostate cancer incidence were conducted in the CPS-II Nutrition Cohort, a subgroup of CPS-II participants enrolled in 1992 and 1993 and observed for both cancer incidence and mortality. ${ }^{14}$ Both cohorts have been approved by the Emory University Institutional Review Board and are described in greater detail in the following sections.

\section{CPS-II}

CPS-II included nearly 1.2 million women and men from 50 US states, the District of Columbia, and Puerto Rico, nearly all 30 years of age or older at enrollment in 1982. ${ }^{13}$ Participants were enrolled by American Cancer Society volunteers and completed a self-administered questionnaire at enrollment that included information on demographic, medical, and lifestyle factors.

Men in CPS-II were never asked if they had had vasectomies. However, most men enrolled in CPS-II together with their wives as part of identifiable spouse pairs. The women's enrollment questionnaire asked about age at first use of various methods of birth control, including "partner vasectomy." Therefore, the vasectomy status of most men in CPS-II could be determined from information reported by their wives.

Exclusions from CPS-II made for this analysis are shown in Figure 1. Of the 508,213 men available for analysis in CPS-II, we excluded those who reported a history of prostate cancer or a cancer that might have been prostate cancer (unknown cancer, male reproductive cancer, or bone cancer; $\mathrm{n}=12,175)$. In addition, we excluded men who were younger than

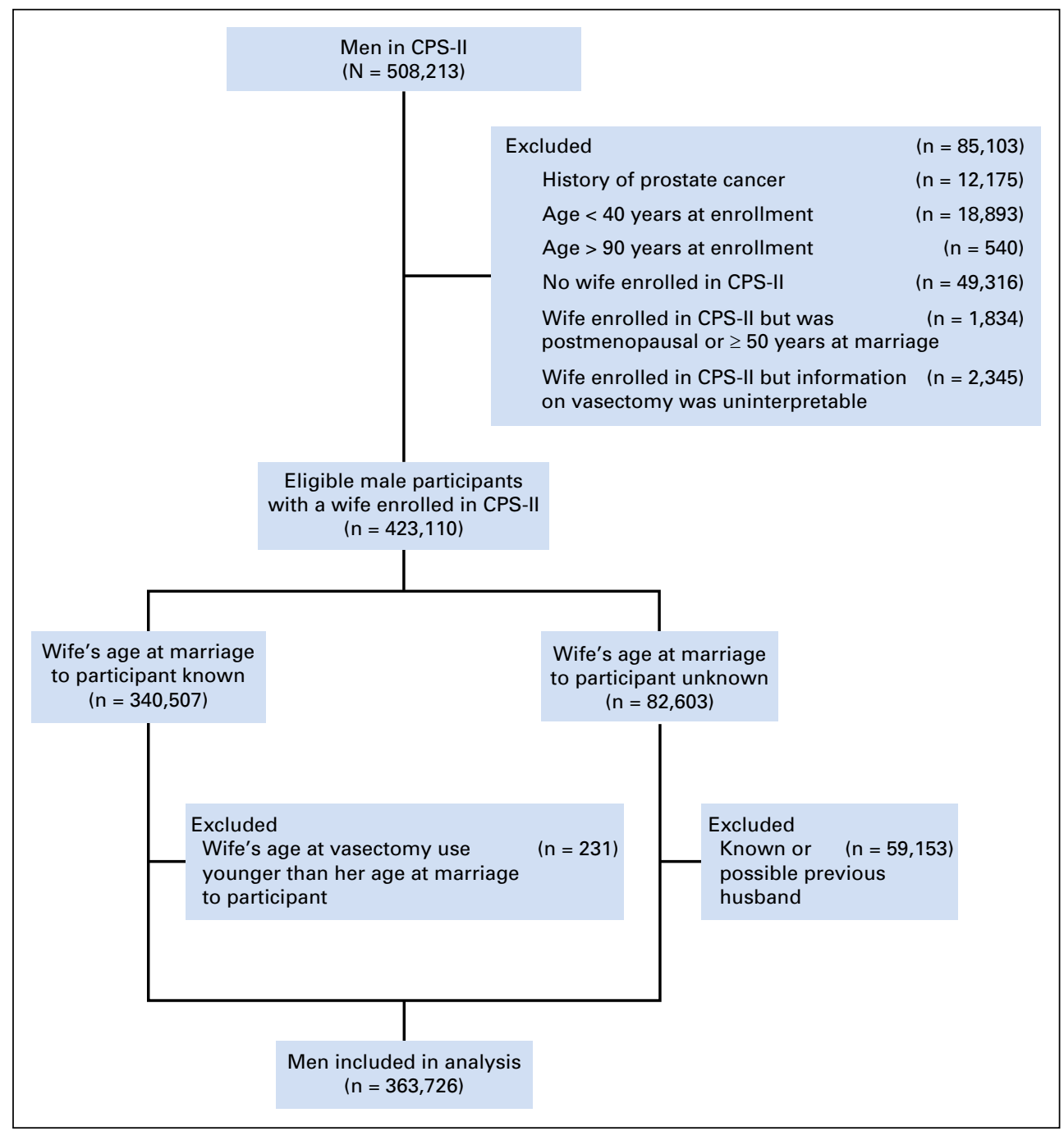

Fig 1. Study population flowchart showing exclusions resulting in a cohort of eligible men with vasectomy status determined from their wives' reports. CPS-II, Cancer Prevention Study II. 
age 40 years at enrollment $(n=18,893)$ and therefore would have been more likely than older men to have vasectomies during follow-up, ${ }^{15}$ as well as men older than age 90 years at enrollment $(n=540)$. We then excluded men whose vasectomy status could not be determined. Specifically, we excluded men who did not have wives in CPS-II in $1982(\mathrm{n}=49,316)$. We also excluded men with wives in CPS-II if their wives were postmenopausal or older than age 50 years at the time of the spouse pairs' marriages ( $\mathrm{n}=1,834)$, because these wives might not have reported their husbands' vasectomies as a method of birth control. We further excluded men whose wives reported uninterpretable or implausible information on vasectomy use $(\mathrm{n}=2,345)$.

We could determine the wife's age at the time of the spouse pair's marriage for each of 340,507 spouse pairs of the remaining 423,110 . We excluded men if their wives' reported first vasectomy use was before the spouse pairs' marriages $(n=231)$. If first vasectomy use occurred after a spouse pair's marriage (ie, during the marriage), we assumed the husband had had a vasectomy.

We could not determine the wives' ages at the time of marriage for 82,603 spouse pairs. In 23,450 of these spouse pairs, the wives reported only one marriage, and we assumed any reported vasectomy referred to their husbands, because vasectomy use before marriage was rarely reported in CPS-II. We excluded men in the remaining spouse pairs $(n=59,153)$, because vasectomy use reported by their wives could have referred to previous husbands. After these exclusions, 363,726 men remained.

A few men reported having had vasectomies on a write-in question asking about history of operations. However, this was uncommon, presumably because vasectomy, an outpatient procedure requiring only local anesthesia, was not always considered an operation. Men who reported vasectomy as a write-in response were categorized as having had vasectomies, even if vasectomy use was not reported by their wives. Of the 42,015 men categorized as having had vasectomies, $86 \%(\mathrm{n}=36,094)$ had the vasectomies reported only by their wives, $12 \%(\mathrm{n}=5,044)$ had the vasectomies reported by their wives and also reported the vasectomies themselves, and $2 \%(\mathrm{n}=877)$ only reported the vasectomies themselves.

Deaths resulting from prostate cancer between enrollment and end of follow-up (December 31, 2012) were ascertained by reviewing death certificates or through linkage with the National Death Index. ${ }^{16}$ Additional information on follow-up for prostate cancer mortality is provided in the Appendix (online only).

\section{CPS-II Nutrition Cohort}

In 1992 and 1993, approximately 10 years after enrollment of the CPS-II cohort, a subset of 184,188 men and women participating in CPS-II from 21 US states were enrolled in the CPS-II Nutrition Cohort. ${ }^{14}$ At enrollment in the Nutrition Cohort, participants completed a selfadministered questionnaire including information on demographic, medical, and lifestyle factors. Follow-up questionnaires to update exposure information and ascertain newly diagnosed cancers were mailed in 1997 and every 2 years thereafter. The response rate for each follow-up questionnaire was at least $86 \%$.

Of the 86,402 men in the Nutrition Cohort, we excluded those who reported a history of prostate cancer either at enrollment in CPS-II or at enrollment in the Nutrition Cohort $(\mathrm{n}=3,729)$. As in the analysis of CPSII, we excluded men who were younger than age 40 years when vasectomy information was collected in $1982(\mathrm{n}=94)$ or whose vasectomy status was uncertain for any of the reasons described under CPS-II $(n=13,506)$. We also excluded those who were alive at the first follow-up questionnaire in 1997 but did not return any follow-up questionnaires $(n=2,372)$ or who reported a prostate cancer on the first follow-up questionnaire that could not be verified $(\mathrm{n}=159)$. After these exclusions, 66,542 men remained. All but 847 of these men were also included in analyses of the parent CPS-II cohort. These 847 men were excluded from analyses of CPS-II, because at enrollment in 1982, they self-reported a history of cancer that might have been prostate cancer (eg, cancer of unknown type), but they could be included in analyses of the Nutrition Cohort, because they reported not having a history of prostate cancer at enrollment in 1992.
This analysis included 9,133 incident prostate cancer cases diagnosed between participant enrollment in 1992 or 1993 and end of follow-up on June 30, 2011. Cases were identified predominantly through participant self-report on biennial follow-up questionnaires and verified with medical records or through linkage with state cancer registries. ${ }^{14,17}$ Additional information on incidence follow-up is provided in the Appendix.

No information on vasectomy status was collected in the CPS-II Nutrition Cohort beyond that collected at enrollment in CPS-II in 1982. Therefore, vasectomy status was defined the same way in analyses of CPS-II and the CPS-II Nutrition Cohort.

\section{Statistical Analyses}

Hazard ratios (HRs) were estimated using Cox proportional hazards and were adjusted for age, race, education level, body mass index, and smoking status, factors associated with prostate cancer mortality in CPS-II. Further adjustment for physical activity, diabetes, family history of prostate cancer, and dietary factors (eg, consumption of eggs, red and processed meats, dairy, fish, and fruit and vegetables) had negligible influence on risk estimates; therefore, these factors were not included in final models. In analyses of prostate cancer incidence, follow-up after 1997 (when information about prostate-specific antigen [PSA] testing was first collected) was adjusted for history of PSA testing using a time-dependent variable for whether PSA testing during the previous follow-up interval had been reported. We adjusted for age using the stratified Cox procedure with 1-year strata; all other variables were modeled using the categories listed in Table 1 . To determine if associations with vasectomy varied, we modeled multiplicative interaction terms between vasectomy and a dichotomous variable for education (with or without a college degree) and continuous variables for attained age and follow-up time.

\section{RESULTS}

All men in our analyses were age 40 years or older at enrollment in CPS-II in 1982. In both cohorts, men with vasectomies tended to be younger than men without, consistent with higher vasectomy rates in more recent birth cohorts. ${ }^{18,19}$ In both cohorts, a great majority of men with or without vasectomies were white (Table 1). In both cohorts, men with vasectomies were slightly more likely to be highly educated and slightly less likely to be obese or current smokers. In the CPS-II Nutrition Cohort, PSA testing (first reported in 1997) was slightly more common among men with vasectomies.

Vasectomy was not associated with prostate cancer mortality in CPS-II (Table 2). Results were similar by years since vasectomy ( $<35$ years since vasectomy: multivariable-adjusted HR, 1.01; 95\% CI, 0.89 to 1.14 ; $\geq 35$ years since vasectomy: multivariableadjusted HR, 1.02; 95\% CI, 0.92 to 1.14 ). No statistically significant interactions were observed between vasectomy and education, attained age, or follow-up time. Results by follow-up year are summarized in Appendix Table Al (online only). Results were similar in analyses restricted to men age 50 years or older when vasectomy status was ascertained in 1982 and therefore less likely to have sought vasectomy during follow-up ${ }^{15}$ (HR, 1.03; 95\% CI, 0.94 to 1.12 ).

Vasectomy was not associated with overall incidence of prostate cancer in the CPS-II Nutrition Cohort (Table 2). Results were similar when examined by years since vasectomy $(<35$ years since vasectomy: multivariable adjusted $\mathrm{HR}, 1.01 ; 95 \% \mathrm{CI}, 0.94$ to 1.08 ; $\geq 35$ years since vasectomy: multivariable-adjusted $\mathrm{HR}, 1.07$; $95 \% \mathrm{CI}, 0.97$ to 1.17 ) and when restricted to men age 50 years or 
Table 1. Selected Characteristics by Vasectomy Status in the CPS-II Cohort

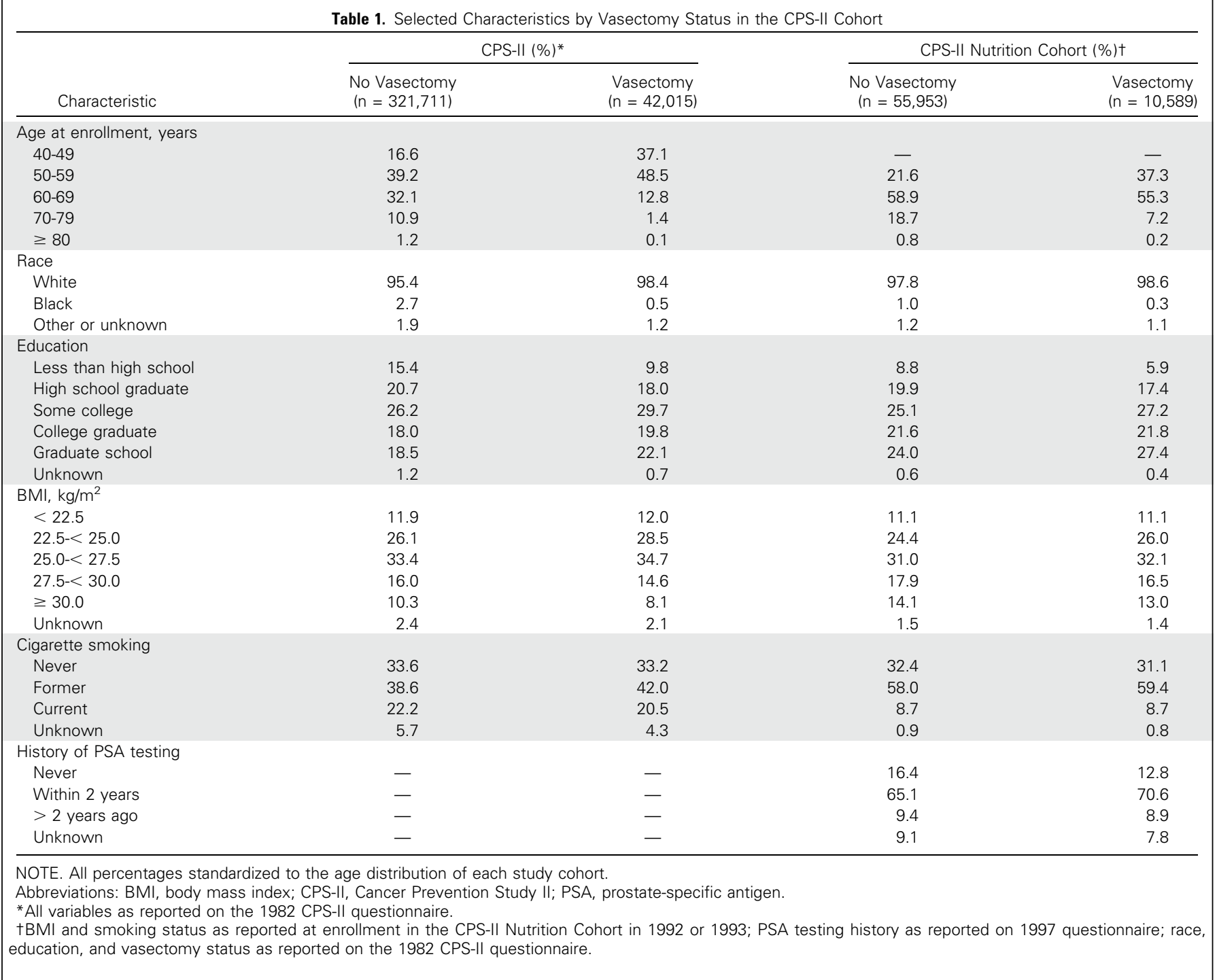

older when vasectomy status was ascertained in 1982 (HR, 0.97; $95 \% \mathrm{CI}, 0.91$ to 1.04 ).

Vasectomy was not associated with risk of prostate cancer of any tumor stage or Gleason score or with aggressive prostate cancer, defined as prostate cancer with high tumor stage and/or high Gleason score at diagnosis (Table 2). The association between vasectomy and prostate cancer incidence did not differ significantly by stage or Gleason score. However, HRs were slightly higher than 1.0 for lower-stage, lower-grade, and nonaggressive cancers, whereas HRs were slightly lower than 1.0 for higher-stage, highergrade, and aggressive prostate cancers. Although prostate cancer mortality was not a focus of Nutrition Cohort analyses, because it was examined with much larger numbers in the overall CPS-II cohort, vasectomy was unexpectedly associated with modestly lower prostate cancer mortality (HR, $0.79 ; 95 \% \mathrm{CI}, 0.64$ to 0.98 , on the basis of 94 cases in vasectomized men). The association between vasectomy and prostate cancer incidence differed by followup time ( $P=.02$ for interaction with continuous follow-up time). Therefore, we examined results by categories of follow-up year
(Appendix Table A2, online only). The HR for vasectomy was close to 1.0 during follow-up through 2006, but it seemed higher during 2007 to 2011, the last 5 years of follow-up (HR, 1.18; 95\% CI, 1.03 to 1.35$)$. The elevated risk observed during 2007 to 2011 was a result of higher risk of nonaggressive prostate cancer (HR, 1.30; $95 \% \mathrm{CI}, 1.11$ to 1.53 ) rather than of aggressive prostate cancer (HR, $0.88 ; 95 \% \mathrm{CI}, 0.65$ to 1.18 ). No statistically significant interactions were observed between vasectomy and education or attained age.

\section{DISCUSSION}

We found no association between vasectomy and prostate cancer mortality in CPS-II, the largest study to date to our knowledge to examine this question. Similarly, in the CPS-II Nutrition Cohort, we found no association of vasectomy with overall prostate cancer incidence or incidence of high-stage or high-grade prostate cancer, although there was an association with higher risk of nonaggressive prostate cancer during the last 5 years of follow-up. 


\begin{tabular}{|c|c|c|c|c|c|c|c|}
\hline \multirow[b]{2}{*}{ Variable } & \multicolumn{3}{|c|}{ No Vasectomy } & \multicolumn{4}{|c|}{ Vasectomy } \\
\hline & No. of Patients & Person-Years & $H R$ & $\begin{array}{l}\text { No. of } \\
\text { Patients }\end{array}$ & Person-Years & $\begin{array}{c}\text { Age- and Race-Adjusted } \\
\text { HR }(95 \% \mathrm{Cl})\end{array}$ & $\begin{array}{c}\text { Multivariable-Adjusted } \\
\text { HR }(95 \% \mathrm{Cl})\end{array}$ \\
\hline \multicolumn{8}{|l|}{ CPS-II* } \\
\hline Prostate cancer mortality & 6,810 & $6,729,115$ & 1.00 (Ref) & 641 & $1,061,622$ & 1.00 (0.92 to 1.09$)$ & $1.01(0.93$ to 1.10$)$ \\
\hline \multicolumn{8}{|l|}{ CPS-II Nutrition Cohort† } \\
\hline $\begin{array}{l}\text { Overall prostate cancer } \\
\text { incidencet }\end{array}$ & 7,587 & 712,378 & 1.00 (Ref) & 1,546 & 147,397 & $1.04(0.98$ to 1.1$)$ & 1.02 (0.96 to 1.08$)$ \\
\hline \multicolumn{8}{|l|}{ By tumor stage $\neq$} \\
\hline T1-T2, No, M0 & 6,482 & 712,378 & 1.00 (Ref) & 1,346 & 147,397 & 1.05 (0.99 to 1.11$)$ & 1.03 (0.97 to 1.09$)$ \\
\hline$\geq \mathrm{T} 3$ or $\mathrm{N} 1$ or $\mathrm{M} 1$ & 790 & 712,378 & 1.00 (Ref) & 149 & 147,397 & $0.93(0.78$ to 1.11$)$ & $0.93(0.78$ to 1.11$)$ \\
\hline \multicolumn{8}{|l|}{ By Gleason scoreł } \\
\hline$\leq 7$ & 5,305 & 712,378 & 1.00 (Ref) & 1,203 & 147,397 & 1.10 (1.04 to 1.18$)$ & 1.08 (1.01 to 1.15$)$ \\
\hline 8-10 & 1,070 & 712,378 & 1.00 (Ref) & 180 & 147,397 & 0.91 (0.78 to 1.07$)$ & $0.91(0.78$ to 1.07$)$ \\
\hline \multicolumn{8}{|l|}{$\begin{array}{l}\text { By aggressive } \\
\text { characteristics } \ddagger \S\end{array}$} \\
\hline Nonaggressive & 4,888 & 712,378 & 1.00 (Ref) & 1,106 & 147,397 & 1.10 (1.03 to 1.18$)$ & 1.08 (1.01 to 1.15$)$ \\
\hline Aggressive & 1,643 & 712,378 & 1.00 (Ref) & 297 & 147,397 & 0.97 (0.84 to 1.08$)$ & $0.95(0.84$ to 1.08$)$ \\
\hline \multicolumn{8}{|c|}{$\begin{array}{l}\text { Abbreviations: CPS-II, Cancer Prevention Study II; HR, hazard ratio; Ref, referent. } \\
\text { *Includes } 363,726 \text { men from the CPS-II cohort observed for mortality from } 1982 \text { to } 2012 \text {. All HRs adjusted for age, race, education, body mass index, and smoking. } \\
\text { tIncludes } 66,542 \text { men from the CPS-II Nutrition Cohort, a subset of the CPS-II cohort observed for incidence from } 1992 \text { to } 2011 \text {. All HRs adjusted for age, race, } \\
\text { education, body mass index, smoking, and history of prostate-specific antigen testing. } \\
\text { łUnclassifiable patient cases were censored at diagnosis date. } \\
\text { §Nonaggressive defined as T1-T2, N0, M0 and Gleason score } \leq 7 \text { at diagnosis. Aggressive defined as T3-T4 or N1 or M1 or Gleason } \geq 8 \text { at diagnosis or fatal prostate } \\
\text { cancer of unknown tumor stage and grade at diagnosis }(n=76) \text {. }\end{array}$} \\
\hline
\end{tabular}

Our results differ somewhat from those of the HPFS, ${ }^{9}$ the largest previous prospective study. In the HPFS, vasectomy was associated with $10 \%$ higher risk of overall prostate cancer (HR, $1.10 ; 95 \%$ CI, 1.04 to 1.17 ) and approximately $20 \%$ higher risk of high-grade prostate cancer (HR, 1.22; 95\% CI, 1.03 to 1.45) and lethal prostate cancer (HR, 1.19; 95\% CI, 1.00 to 1.43). Differing results between our study and the HPFS could have resulted from chance. The associations of vasectomy with prostate cancer mortality and prostate cancer incidence in our study were not statistically significantly different from those in the HPFS $(P=.10$ for prostate cancer mortality; $P=.08$ for incidence). It is possible that a modest association with risk of aggressive or fatal prostate cancer in our study population could have been obscured by residual confounding if vasectomized men were substantially more likely than men without vasectomies to have had prostate cancer detected earlier. However, in our cohort, reported rates of PSA testing were only slightly higher among men with vasectomies. Adjustment for education and history of PSA testing, likely correlates of earlier detection, had little influence on results, although self-reported PSA testing may be inaccurate, and residual confounding cannot be ruled out. The absence of an association between vasectomy and prostate cancer incidence in the CPS-II Nutrition Cohort cannot be explained by earlier detection among men with vasectomies. Future studies in populations where PSA screening is not used, or where detailed information on potential predictors of early detection are available, including income and health insurance coverage and use, may be helpful in addressing potential residual confounding.

The association between vasectomy and prostate cancer incidence in our study differed by follow-up time $(P=.02)$, with higher risk of diagnosis with nonaggressive prostate cancer among vasectomized men during the final years of follow-up (2007 to 2011). Reasons for this difference are unclear. Guidelines recommending against PSA screening for men age older than 75 years of age were released in 2008 and might have influenced association with nonaggressive prostate cancer. ${ }^{20}$ However, we did not observe a divergence in self-reported PSA testing by vasectomy status after 2008. On the 1997 questionnaire, the first time we asked about PSA testing, the age-adjusted prevalence of a PSA test within the previous 2 years was $71 \%$ among men with vasectomies and $65 \%$ among men without. On the final two questionnaires, the corresponding prevalences were $79 \%$ and $76 \%$ (in 2009) and 75\% and $72 \%$ (in 2011). Chance may have contributed to the observed differences over time.

Important strengths of this analysis include its prospective design and large size. This analysis included more than 7,000 deaths resulting from prostate cancer, compared with just more than 800 in the HPFS, the next largest study of vasectomy and prostate cancer mortality. ${ }^{9}$

A limitation of this analysis is that vasectomy status was reported by participants' wives, potentially resulting in some underreporting. However, the sensitivity of the wives' reports of vasectomy use seemed relatively high. Of the 5,891 vasectomies reported as a write-in response by husbands, $85 \%(\mathrm{n}=5,044)$ were also reported by their wives. Misclassification of vasectomy status could also have resulted from vasectomies received after enrollment, because information on vasectomy status was not updated. However, it is unlikely vasectomies after enrollment were common. When vasectomy status was reported at enrollment in 1982, all men in our analysis were already age 40 years or older, and $96 \%$ of men in the Nutrition Cohort and $97 \%$ of men in the overall CPS-II cohort were already age 45 years or older. Most men never undergo vasectomy, and those who do typically have one before reaching middle age. In a study from the 1990s designed to be nationally representative, only $21 \%$ of vasectomized patients were age 40 years or older (whereas more than $50 \%$ of all US men were 
age $>40$ years), and only $7 \%$ were age 45 years or older. ${ }^{15}$ Our results were also similar when analyses were restricted to men age older than 50 years at enrollment, well past the typical age of vasectomy.

It is important to note that misclassification of some men who had vasectomies as not having had them seems unlikely to have meaningfully biased our results. Such misclassification would have had little effect on the relative risk for vasectomy, because these men could plausibly have constituted only a small proportion of all men categorized as not having had vasectomies. The expected amount of bias resulting from varying amounts of misclassification is explained in detail in Appendix Figure A1 (online only). We estimate that even if $5 \%$ of the overall cohort actually had vasectomies but was misclassified as not having had them (ie, the true prevalence of vasectomy was $17 \%$ instead of the $12 \%$ reported), and vasectomy were associated with a $20 \%$ increase in prostate cancer mortality (as in the HPFS), the rate ratio for vasectomy would only have been underestimated by approximately $1 \%$.

Results from this large prospective study do not support an association between vasectomy and either prostate cancer incidence or mortality. Because this study, like all observational studies, has some potential for bias, a small increase in risk cannot be entirely ruled out. However, our results provide some reassurance that vasectomy is unlikely to meaningfully increase risk of prostate cancer.

\section{AUTHORS' DISCLOSURES OF POTENTIAL CONFLICTS} OF INTEREST

Disclosures provided by the authors are available with this article at www.jco.org.

\section{AUTHOR CONTRIBUTIONS}

Conception and design: Eric J. Jacobs, Rebecca L. Anderson

Collection and assembly of data: Eric J. Jacobs, Susan M. Gapstur

Data analysis and interpretation: All authors

Manuscript writing: All authors

Final approval of manuscript: All authors

\section{REFERENCES}

1. Sharlip ID, Belker AM, Honig S, et al: Vasectomy: AUA guideline. J Urol 188:2482-2491, 2012 (suppl)

2. Dennis LK, Dawson DV, Resnick MI: Vasectomy and the risk of prostate cancer: A meta-analysis examining vasectomy status, age at vasectomy, and time since vasectomy. Prostate Cancer Prostatic Dis 5:193-203, 2002

3. Giovannucci E, Ascherio A, Rimm EB, et al: A prospective cohort study of vasectomy and prostate cancer in US men. JAMA 269:873-877, 1993

4. Giovannucci E, Tosteson TD, Speizer FE, et al: A retrospective cohort study of vasectomy and prostate cancer in US men. JAMA 269: 878-882, 1993

5. Rohrmann S, Paltoo DN, Platz EA, et al: Association of vasectomy and prostate cancer among men in a Maryland cohort. Cancer Causes Control 16: 1189-1194, 2005

6. Sidney S, Quesenberry CP Jr, Sadler MC, et al: Vasectomy and the risk of prostate cancer in a cohort of multiphasic health-checkup examinees: Second report. Cancer Causes Control 2:113-116, 1991
7. Lynge $E$ : Prostate cancer is not increased in men with vasectomy in Denmark. J Urol 168: 488-490, 2002

8. Goldacre MJ, Wotton CJ, Seagroatt $V$, et al: Cancer and cardiovascular disease after vasectomy: An epidemiological database study. Fertil Steril 84: 1438-1443, 2005

9. Siddiqui MM, Wilson KM, Epstein MM, et al: Vasectomy and risk of aggressive prostate cancer: $\mathrm{A}$ 24-year follow-up study. J Clin Oncol 32:3033-3038, 2014

10. Pereira $S$, Martinez M, Martinez FE, et al: Repercussions of castration and vasectomy on the ductal system of the rat ventral prostate. Cell Biol Int 30:169-174, 2006

11. Howards SS: Possible biological mechanisms for a relationship between vasectomy and prostatic cancer. Eur J Cancer 29A:1060-1062, 1993

12. Scovell JM, Wilken N, Ramasamy $R$, et al: Words of wisdom: Re: Vasectomy and risk of aggressive prostate cancer-A 24-year follow-up study. Eur Urol 66:1186-1187, 2014

13. Stellman SD, Garfinkel L: Smoking habits and tar levels in a new American Cancer Society prospective study of 1.2 million men and women. J Natl Cancer Inst 76:1057-1063, 1986
14. Calle EE, Rodriguez C, Jacobs EJ, et al: The American Cancer Society Cancer Prevention Study II nutrition cohort: Rationale, study design, and baseline characteristics. Cancer 94:2490-2501, 2002

15. Barone MA, Johnson $\mathrm{CH}$, Luick MA, et al: Characteristics of men receiving vasectomies in the United States, 1998-1999. Perspect Sex Reprod Health 36:27-33, 2004

16. Calle EE, Terrell DD: Utility of the National Death Index for ascertainment of mortality among cancer prevention study II participants. Am J Epidemiol 137:235-241, 1993

17. Bergmann MM, Calle EE, Mervis CA, et al: Validity of self-reported cancers in a prospective cohort study in comparison with data from state cancer registries. Am J Epidemiol 147:556-562, 1998

18. Mosher WD, Westoff CF: Trends in contraceptive practice: United States, 1965-76. Vital Health Stat 23 10:1-47, 1982

19. Mosher WD, Pratt WF: Contraceptive use in the United States, 1973-88. Patient Educ Couns 16: 163-172, 1990

20. U.S. Preventive Services Task Force: Screening for prostate cancer: U.S. Preventive Services Task Force recommendation statement. Ann Intern Med 149:185-191, 2008 


\section{AUTHORS' DISCLOSURES OF POTENTIAL CONFLICTS OF INTEREST}

Vasectomy and Prostate Cancer Incidence and Mortality in a Large US Cohort

The following represents disclosure information provided by authors of this manuscript. All relationships are considered compensated. Relationships are self-held unless noted. I = Immediate Family Member, Inst = My Institution. Relationships may not relate to the subject matter of this manuscript. For more information about ASCO's conflict of interest policy, please refer to www.asco.org/rwc or jco.ascopubs.org/site/ifc.

\section{Eric J. Jacobs}

No relationship to disclose

Rebecca L. Anderson

No relationship to disclose

Victoria L. Stevens

No relationship to disclose

\section{Christina C. Newton}

No relationship to disclose

Ted Gansler

No relationship to disclose

Susan M. Gapstur

No relationship to disclose 


\section{Acknowledgment}

We acknowledge the contribution to this study from central cancer registries supported through the Centers for Disease Control and Prevention National Program of Cancer Registries, as well as cancer registries supported by the National Cancer Institute SEER program.

\section{Appendix}

To ascertain the vital status of Cancer Prevention Study II (CPS-II) participants, American Cancer Society volunteers made personal inquiries in September 1984, 1986, and 1988 to determine whether the participants enrolled were alive or dead and recorded the dates and places of deaths. Reported deaths were verified by obtaining death certificates. At completion of the 1988 follow-up, vital status was known for $98.2 \%$ of the cohort. Linkage to the National Death Index (NDI) was used to identify deaths that occurred between September 1988 and December 2012 and to identify deaths among 21,704 participants lost to follow-up between 1982 and 1988. ${ }^{16}$ Death certificates or codes for causes of death were obtained for more than $99 \%$ of all known deaths. Mortality follow-up was truncated at age 90 years in this analysis, because a small percentage of deaths are missed by NDI linkage, ${ }^{16}$ which could result in significant misclassification of vital status at advanced ages, when a high proportion of participants are dead. The underlying cause of death was defined according to the International Classification of Diseases, Ninth Revision (ICD-9; World Health Organization: International Classification of Diseases: Ninth Revision. Geneva, Switzerland, World Health Organization, 1977) for deaths occurring from 1992 through 1998 and the 10th revision (ICD-10; World Health Organization: International Statistical Classification of Diseases and Related Health Problems: Tenth Revision. Geneva, Switzerland, World Health Organization, 1992) for deaths occurring from 1999 through 2012. Deaths resulting from prostate cancer were defined as ICD-9 code 185 or as ICD-10 code C61. No separate medical record reviews to ascertain cause of death were performed. Agreement between medical record review and death certificates is reasonably good for prostate cancer mortality (Albertsen PC, et al: J Urol 163:519523, 2000; Hoffman RM, et al: Ann Epidemiol 13:450-454, 2003; Penson DF, et al: J Natl Cancer Inst 93:1822-1823, 2001; Barry MJ, et al: Clin Trials 10:907-914, 2013).

Of the 9,133 incident prostate cancer cases included in analyses of the CPS-II Nutrition Cohort, 8,833 were initially identified by self-report on the follow-up questionnaires and then verified by obtaining medical records or through linkage with state cancer registries when medical records could not be obtained. ${ }^{14}$ Ascertainment of cancer by self-report is estimated to have a sensitivity of 93\% in the Nutrition Cohort. ${ }^{17}$ Nutrition Cohort participants, as members of the larger CPS-II cohort, were also observed for mortality through linkage with the NDI. An additional 300 fatal cases of prostate cancer, not identified through self-report, were identified through linkage with the NDI, ${ }^{16}$ of which 198 were verified through subsequent linkage with state cancer registries. All incident prostate cancers (ICD-9 code 185 or ICD-10 code C61) were included as outcomes, except for a small number that were coded as papillary serous cystadenocarcinomas or as specific histologic types that were not adenocarcinomas.

\begin{tabular}{|c|c|c|c|c|c|c|}
\hline \multirow[b]{2}{*}{ Follow-Up Period } & \multicolumn{3}{|c|}{ No Vasectomy } & \multicolumn{3}{|c|}{ Vasectomy } \\
\hline & No. of Patients & Person-Years & $\mathrm{HR}$ & No. of Patients & Person-Years & HR $(95 \% \mathrm{Cl})$ \\
\hline 1982-1991 & 1,336 & $2,785,705$ & 1.00 (Ref) & 52 & 379,985 & $0.79(0.60$ to 1.05$)$ \\
\hline 1992-2001 & 2,844 & $2,353,193$ & 1.00 (Ref) & 209 & 367,562 & $0.97(0.84$ to 1.12$)$ \\
\hline $2002-2012$ & 2,630 & $1,590,159$ & 1.00 (Ref) & 380 & 314,072 & $1.07(0.96$ to 1.20$)$ \\
\hline$P$ trend ${ }^{*}$ & & & & & & .18 \\
\hline
\end{tabular}


Table A2. Prostate Cancer Incidence by Vasectomy Status and Calendar Follow-Up Period in the CPS-II Nutrition Cohort

\begin{tabular}{|c|c|c|c|c|c|c|}
\hline \multirow[b]{2}{*}{ Follow-Up Period } & \multicolumn{3}{|c|}{ No Vasectomy } & \multicolumn{3}{|c|}{ Vasectomy } \\
\hline & No. of Patients & Person-Years & $H R$ & No. of Patients & Person-Years & $\mathrm{HR}(95 \% \mathrm{Cl})$ \\
\hline \multicolumn{7}{|l|}{ Overall prostate cancer incidence } \\
\hline 1992-1996 & 1,970 & 207,873 & 1.00 (Ref) & 307 & 40,707 & 0.95 (0.84 to 1.07$)$ \\
\hline $1997-2001$ & 2,781 & 224,651 & 1.00 (Ref) & 536 & 44,863 & $1.01(0.92$ to 1.11$)$ \\
\hline 2002-2006 & 1,944 & 170,053 & 1.00 (Ref) & 433 & 36,323 & $1.01(0.91$ to 1.12$)$ \\
\hline 2007-2011 & 892 & 109,800 & 1.00 (Ref) & 270 & 25,504 & $1.18(1.03$ to 1.35$)$ \\
\hline$P$ trend* & & & & & & .02 \\
\hline \multicolumn{7}{|c|}{ Aggressive prostate cancer incidence ${ }^{\prime}$} \\
\hline 1992-1996 & 487 & 207,873 & 1.00 (Ref) & 63 & 40,707 & $0.78(0.60$ to 1.02$)$ \\
\hline $1997-2001$ & 537 & 224,651 & 1.00 (Ref) & 101 & 44,863 & 1.02 (0.82 to 1.26$)$ \\
\hline $2002-2006$ & 359 & 170,053 & 1.00 (Ref) & 80 & 36,323 & $1.13(0.88$ to 1.45$)$ \\
\hline $2007-2011$ & 260 & 109,800 & 1.00 (Ref) & 53 & 25,504 & 0.88 (0.65 to 1.18$)$ \\
\hline$P$ trend* & & & & & & .14 \\
\hline \multicolumn{7}{|c|}{ Nonaggressive prostate cancer incidenceł } \\
\hline 1992-1996 & 1,122 & 207,873 & 1.00 (Ref) & 188 & 40,707 & $0.98(0.84$ to 1.15$)$ \\
\hline $1997-2001$ & 1,779 & 224,651 & 1.00 (Ref) & 378 & 44,863 & $1.07(0.96$ to 1.20$)$ \\
\hline $2002-2006$ & 1,383 & 170,053 & 1.00 (Ref) & 327 & 36,323 & $1.03(0.91$ to 1.16$)$ \\
\hline $2007-2011$ & 604 & 109,800 & 1.00 (Ref) & 213 & 25,504 & 1.30 (1.11 to 1.53$)$ \\
\hline$P$ trend $^{*}$ & & & & & & .07 \\
\hline
\end{tabular}

NOTE. Includes 66,542 men from the CPS-II Nutrition Cohort, a subset of the CPS-II cohort, observed for incidence from 1992 to 2011 . All analyses adjusted for age, race, education, body mass index, smoking, and history of prostate-specific antigen testing.

Abbreviations: CPS-II, Cancer Prevention Study II; HR, hazard ratio; Ref, referent.

*Calculated using a continuous variable for time since enrollment.

tDefined as $\geq \mathrm{T} 3$ or N1 or M1 or Gleason $\geq 8$ at diagnosis or fatal prostate cancer of unknown tumor stage and grade at diagnosis ( $\mathrm{n}=76$ ). Prostate cancer cases with insufficient information to determine aggressiveness were censored.

‡Defined as T1-T2, N0, or MO and Gleason score $\leq 7$ at diagnosis. Prostate cancer cases with insufficient information to determine aggressiveness were censored.

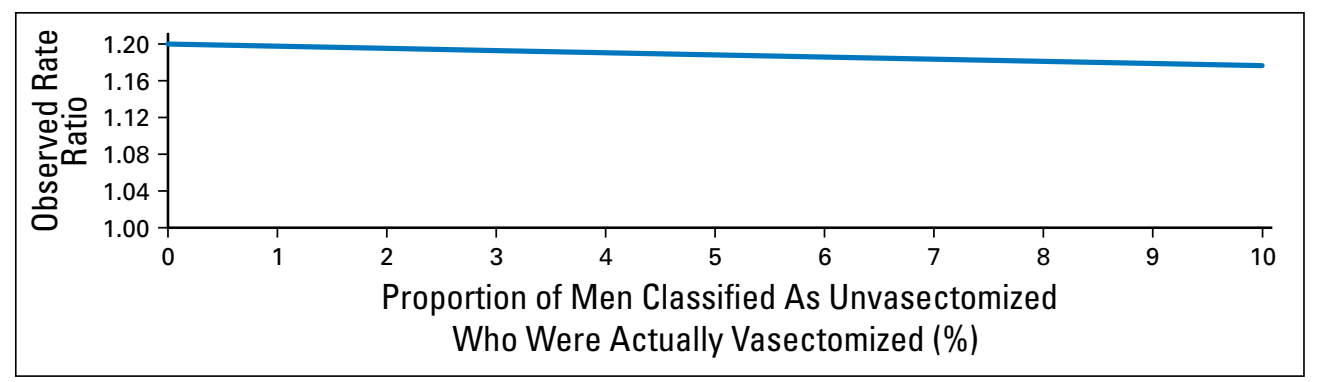

Fig A1. Misclassification of vasectomy status could have occurred because of under-reporting of vasectomy at enrollment and missed vasectomies after enrollment. The figure illustrates the observed rate ratio as a function of the proportion of men classified as unvasectomized who were actually vasectomized. The form of this function is justified in the following equations. The observed rate ratio comparing vasectomized versus unvasectomized men is: (Rate observed in men classified as vasectomized)/ (Rate observed in men classified as unvasectomized). If the true rate of prostate cancer mortality is $\mathrm{M}$ in unvasectomized men, and 1.20M in vasectomized men (based on the rate ratio of 1.20 observed in the Health Professional Follow-Up Study), $x$ is the proportion of men classified as unvasectomized who were actually vasectomized, and a weighted average is used to calculate the rate in the denominator in terms of $\mathrm{M}$, then the observed rate ratio is: $1.20 /[(1-x)(1 \mathrm{M})+\mathrm{x}(1.20 \mathrm{M})]$. This expression simplifies to $1.20 /(1+0.20 x)$, which is plotted in the figure. Attenuation of the true rate ratio is relatively modest even when $10 \%$ of men classified as unvasectomized were actually vasectomized. 